\title{
ENVIRONMENTAL SENSING BY AFRICAN TRYPANOSOMES
}

Isabel Roditi*, Gabriela Schumann \& Arunasalam Naguleswaran

Institute for Cell Biology, University of Bern, Bern, Switzerland.

*Corresponding author: isabel.roditi@izb.unibe.ch

Abstract

African trypanosomes, which divide their life cycle between mammals and tsetse flies, are confronted with environments that differ widely in temperature, nutrient availability and host responses to infection. In particular, since trypanosomes cannot predict when they will be transmitted between hosts, it is vital for them to be able to sense and adapt to their milieu. Thanks to technical advances, significant progress has been made in understanding how the parasites perceive external stimuli and react to them. There is also a growing awareness that trypanosomes use a variety of mechanisms to exchange information with each other, thereby enhancing their chances of survival. 


\section{Introduction}

Trypanosoma brucei rhodesiense and T. b. gambiense, the parasites that cause human sleeping sickness, and their close relative $T$. b. brucei, which infects animals but not humans, cycle between mammals and tsetse flies. These two hosts provide very different environments as the parasites progress through their life cycle. Some of these differences are fixed and host-/tissue-specific, while others can vary depending on the ambient temperature, nutritional status and immune response to infection. Trypanosomes are able to sense different features of their surroundings and to adapt accordingly. Some adaptations, such as the response to heat stress, are reversible, while others, such as differentiation to the next life-cycle stage, are irreversible. Although it has been known for decades that trypanosomes sense their surroundings, it is only in the past few years that significant headway has been made in understanding the molecules and pathways that underlie this.

\section{Sensing host identity}

Of the three sub-species, $T$. $b$. rhodesiense has the largest host range, as it can infect humans and a wide variety of animals. Bloodstream forms take up host serum components and, in the process, can "identify" their host. The variant surface glycoprotein that forms the parasite's surface coat, and several other proteins, including the transferrin receptor, are encoded in the bloodstream form expression site (BES). Trypanosomes have 10-15 versions of the BES, but only one is transcribed in an individual cell. There are several variants of the transferrin receptor that differ in their affinities for transferrin from different species [1]. Thus, cells that express a high affinity receptor for the species they infect, or cells that switch to the BES encoding this receptor, have a growth advantage [1]. Trypanosomes also take up high-density lipoprotein (HDL), a source of heme, via the haptoglobin-hemoglobin receptor (TbHpHbR) [2]. HDL differs in composition depending on the host. In humans, a subset of HDL contains apolipoprotein LI (APOLI) [3], which kills T. $b$. brucei by creating pores in the mitochondrion [ $\left.4^{* *}\right]$. Both T. b. rhodesiense and T. $b$. gambiense have specific proteins that neutralize the effects of APOLI [5-7]. The SRA protein from $T . b$. rhodesiense is encoded in a single BES, and when trypanosomes are grown in the presence of human serum they activate its expression [5]. Under 
these circumstances, one would expect that parasites that switch to another BES in humans would be killed. Recently, it was shown that bloodstream forms release exosomes from the flagellum and the flagellar pocket and that these can transfer proteins (including SRA) to other trypanosomes in culture, rendering them less sensitive to lysis $\left[8^{*}\right]$. It still needs to be established, however, if exosomes are capable of transferring sufficient SRA to protect parasites against APOLI in vivo. In contrast to T. $b$. rhodesiense, T. b. gambiense has a mutation in TbHpHbR that limits the uptake of HDL $[6,9]$ and its APOLI-neutralizing protein, TgsGP, is constitutively expressed [6]. These adaptations may come at a price, however, since its host range is more limited.

\section{Quorum sensing in the mammal}

In mammals, trypanosomes detect each other's presence by quorum sensing and react by balancing the proportions of two different life-cycle stages [10]. This is essential for prolonging the infection and maximizing the chances of transmission to the tsetse fly. Stumpy induction factor (SIF) is a low molecular mass molecule (or mixture of molecules) released by proliferating slender bloodstream forms [11]. When SIF reaches a critical threshold, it drives differentiation of slender forms to non-dividing stumpy forms. This has the dual effect of capping the parasite titer, which might otherwise result in the death of the host, and providing a continuous supply of transmissible forms. A number of genes have been implicated in the differentiation of slender to stumpy forms. Three kinases (MAPK5, ZFK and TOR4) increase the threshold required for the activity of SIF, thereby preventing premature differentiation at low parasite densities [12-14]. It is not known if these kinases form part of a cascade or if they act independently. Genome-wide RNAi screens for trypanosomes that are resistant to SIF mimetics (cell-permeable analogues of cAMP or AMP), have been powerful tools for identifying genes required for differentiation to stumpy forms $\left[15^{* *}, 16\right]$. These include the RNA-binding protein RBP7, several kinases and phosphatases, and a number of hypothetical proteins whose functions still need to be elucidated. Since the compounds used in these screens can cross the plasma membrane, the SIF receptor is unlikely to be among the genes identified. 


\section{Integrating environmental cues for differentiation}

When trypanosomes are ingested by a tsetse fly, stumpy forms differentiate into procyclic forms in the insect midgut. Parasites can also be triggered to differentiate in vitro in the presence of citrate/ cis-aconitate (CCA) [17], which is probably the physiological signal in vivo as well [18]. A crucial part of the differentiation signal is the drop in temperature. At $37^{\circ} \mathrm{C}$, differentiation requires extremely high concentrations of CCA and, although the cells begin to express the midgut-specific surface protein EP procyclin, they do not proliferate. At $20^{\circ} \mathrm{C}$, however, trypanosomes become hypersensitive to CCA, differentiation is completed and the parasites re-enter the cell cycle. PAD2 is a member of a family of carboxylate transporters that is expressed in stumpy forms, but not in slender forms. Both its expression and localization are thermo-sensitive. Decreasing the temperature from $37^{\circ} \mathrm{C}$ to $20^{\circ} \mathrm{C}$ increases steady-state levels of PAD2 protein approximately 4 -fold and results in it moving from the flagellar pocket to the cell surface. Although it is not known if other proteins are thermo-sensitive, one candidate is repressor of differentiation kinase 1 (RDK1) [19**]. Depletion of RDK1 by RNA interference results in the expression of EP procyclin at $37^{\circ} \mathrm{C}$, in the absence of $C C A$, and this effect is potentiated at $27^{\circ} \mathrm{C}$. Two other proteins also function as differentiation repressors, the phosphatase TbPTP1 [20] and RDK2 [19**], but these do not appear to be temperature-dependent [19**]. Recently, a screen for compounds that trigger differentiation identified several small molecules unrelated to CCA that triggered expression of procyclic markers at $37^{\circ} \mathrm{C}[21]$. One possibility is that these are inhibitors of TbPTP1, the RDKs or their downstream targets.

\section{Reacting to heat stress}

Trypanosomes in the fly are confronted with much larger fluctuations in temperature than the bloodstream forms in the mammal, and must be able to survive heat shock as well as cold shock. Exposure of procyclic forms to severe heat shock has wide-ranging effects on transcription, mRNA processing and translation $[22,23]$. Two proteins have been implicated in resistance to heat stress, the kinase MAPK4 [24] and the zinc finger protein ZC3H11 [25,26*]. It has been shown that the 
latter binds preferentially to chaperone-encoding mRNAs and stabilizes them [25]. ZC3H11 itself responds to heat shock and accumulates at higher temperatures. This is partly due to an increase in its stability and partly due to more efficient translation [26*]. The effect on translation is mediated by an element in the $3^{\prime}$ untranslated region (UTR) that appears to be a binding site for a repressor at lower temperatures. Another effect of severe heat shock is that nuclear DNA becomes more tightly associated with histones $\left[27^{*}\right]$. Increased histone occupancy coincides with the translocation of the chromatin-associated protein TbRRM1 to the cytoplasm; when cells are returned to their normal temperature TbRRM1 relocates to the nucleus. Depletion of TbRRM1 by RNA interference also increases histone occupancy, suggesting this protein is mediating the response $\left[27^{*}\right]$.

\section{Cross-talk between mRNAs and metabolites}

The stability of several mRNAs in procyclic trypanosomes is affected by metabolites in the environment. GPEET procyclin, the major surface protein of newly differentiated procyclic forms [28], is expressed for the first 4-7 days in the tsetse midgut, and repressed thereafter. In culture, GPEET mRNA is stabilized in the presence of glycerol and destabilized in the presence of glucose or under hypoxic conditions $[28,29]$. A single element in the 3' UTR mediates all these responses, as well being responsible for the stage-specific expression of GPEET in tsetse $[28,29]$. Procyclins are transcribed by RNA polymerase I in the nucleolus, together with ribosomal RNAs [30]. A nucleolar complex required for rRNA processing has been implicated in destabilization of GPEET mRNA (either co-transcriptionally or shortly afterwards) [31], but it is not known if its activity is sensitive to metabolites. It is also not known if there are additional levels of regulation of GPEET mRNA stability and translation in the cytoplasm.

Procyclic trypanosomes are able to sense nutrient status and regulate the uptake of precursors. The mRNA encoding the nucleoside transporter TbNT8 is reduced in the early logarithmic phase of growth and accumulates in late log phase [32**]. This response is mediated by a stem-loop in the $3^{\prime}$ UTR of TbNT8 that destabilizes the mRNA in the presence of exogenous purines. It is not known if this is a direct effect of purine nucleosides themselves, in which they act as aptamers, or if 
protein mediators are involved. Approximately 200 other mRNAs are differentially expressed in early and late log parasites $\left[32^{* *}\right]$ and it is possible that many of them are nutritional sensors.

\section{Cell-cell communication by procyclic forms}

When procyclic forms are grown on agarose plates, rather than in liquid medium, they show coordinated movement, a phenomenon termed social motility (SoMo) [33]. Early (GPEET-positive) procyclic forms first proliferate at the inoculation site, but once a critical cell number is reached [34*] communities of trypanosomes migrate outwards together, forming radial projections [33]. This implies that these cells both produce and respond to migration factors. When two sets of parasites are inoculated on the same plate, radial projections from the two communities perceive each other and reorient to avoid contact [33]. This is not simply due to recognition of self and non-self as it also occurs with two communities originating from the same liquid culture. Late (GPEET-negative) procyclic forms do not migrate, but they produce a repellent that is sensed by early procyclic forms [34]. Local production of cyclic AMP regulates this behavior - cells lacking phosphodiesterase B1 are negative for SoMo and do not produce the migration factor [35**], while cells depleted of two adenylate cyclases that localize to the flagellar tip produce more radial projections $\left[36^{*}, 37\right]$. A glycosylation mutant lacking the $R f t 1$ gene is defective in SoMo and is also compromised in its ability to establish midgut infections [38]. Thus, the restriction of SoMo to early procyclic forms might reflect a role early in infection, such as the migration of parasites from the endo- to the ectoperitrophic space.

It was recently discovered that procyclic forms can exchange cell contents directly [39], which should give them an intimate sense of the condition of other trypanosomes in the environment. This occurs by flagellar membrane fusion and the bidirectional transfer of proteins. The process is inhibited by EGTA, indicating a requirement for extracellular calcium [39]. In some cases there is only partial fusion of the flagella, and the cells separate again, while in other cases fusion occurs along the entire length of the flagella and the cells may remain together indefinitely. Pairs of cells with fused flagella are motile and can divide, indicating that fusion is not 
harmful. To date, however, fusion and protein transfer have only been observed in culture, and relatively rarely, and their biological significance is not yet understood.

\section{Quo vadis?}

As shown above, trypanosomes can perceive and react to a wide variety of stimuli (Figure 1). All the differentiation steps that have been studied so far occur in response to external cues, but apart from temperature we have no idea what signals might be delivered by the fly. There is a $\mathrm{pH}$ gradient in the midgut [40] and it is also possible that the various compartments differ in osmotic pressure, ionic strength and availability of nutrients. At present, however, there is no information on whether these are sensed by the parasites or if they have an impact on differentiation. RBP6 is an RNA-binding protein that is up-regulated in (some) trypanosomes in the proventriculus [41]. Ectopic expression of RBP6 in procyclic forms suffices to drive their differentiation to epimastigote and metacyclic forms in culture [41]. It is not known what regulates RBP6 expression in vivo, but it would not be surprising if its 3' UTR acts as an environmental sensor.

A recurring theme in this review is that we have pieces of various puzzles, but are far from having a complete picture of any one pathway. Another theme is the extent of parasite communication, which was vastly underestimated until very recently, and which needs to be put into its biological context. There is still much waiting to be discovered, such as the identity of SIF or the factors involved in SoMo. Recent technical developments, such as increased transfection efficiencies [42], genome-wide screens $[15,31]$, high throughput sequencing $[25,41]$ and increasingly sensitive mass spectrometry underlie many of the discoveries mentioned in this article and, in all likelihood, metabolomics will have an impact on the field in the near future. All in all, we live in exciting times for this growing area of research.

\section{ACKNOWLEDGMENTS}

Work in our laboratory is supported by the Canton of Bern, the Swiss National Science Foundation (grant no. 31003A-144142) and the Howard Hughes Medical Institute International Senior Scholars Program (grant no. 55007650). We thank 
Sebastian Millius for providing an image of a SoMo plate and Kent Hill for discussions during an early phase of drafting the manuscript. 


\section{REFERENCES}

1. Bitter $W$, Gerrits $H$, Kieft $R$, Borst $P$ : The role of transferrin-receptor variation in the host range of Trypanosoma brucei. Nature 1998, 391:499-502.

2. Vanhollebeke B, De Muylder G, Nielsen MJ, Pays A, Tebabi P, Dieu M, Raes M, Moestrup SK, Pays E: A haptoglobin-hemoglobin receptor conveys innate immunity to Trypanosoma brucei in humans. Science 2008, 320:677-681.

3. Vanhamme $L$, Pays $E$ : The trypanosome lytic factor of human serum and the molecular basis of sleeping sickness. Int J Parasitol 2004, 34:887-898.

4. ${ }^{* *}$ Vanwalleghem G, Fontaine F, Lecordier L, Tebabi P, Klewe K, Nolan DP, Yamaryo-Botte Y, Botte C, Kremer A, Schumann Burkard G, et al.: Coupling of lysosomal and mitochondrial membrane permeabilization in trypanolysis by APOL1. Nat Commun 2015, 6:8078.

A genome-wide screen identified a key parasite component contributing to lysis by APOLI and elucidated the mechanism of killing.

5. Xong HV, Vanhamme L, Chamekh M, Chimfwembe CE, Van Den Abbeele J, Pays A, Van Meirvenne N, Hamers R, De Baetselier P, Pays E: A VSG expression siteassociated gene confers resistance to human serum in Trypanosoma rhodesiense. Cell 1998, 95:839-846.

6. Uzureau P, Uzureau S, Lecordier L, Fontaine F, Tebabi P, Homble F, Grelard A, Zhendre V, Nolan DP, Lins L, et al.: Mechanism of Trypanosoma brucei gambiense resistance to human serum. Nature 2013, 501:430-434.

7. Capewell P, Clucas C, DeJesus E, Kieft R, Hajduk S, Veitch N, Steketee PC, Cooper A, Weir W, MacLeod A: The TgsGP gene is essential for resistance to human serum in Trypanosoma brucei gambiense. PLoS Pathog 2013, 9:e1003686.

8.* Szempruch AJ, Sykes SE, Kieft R, Dennison L, Becker AC, Gartrell A, Martin WJ, Nakayasu ES, Almeida IC, Hajduk SL, et al.: Extracellular Vesicles from Trypanosoma brucei Mediate Virulence Factor Transfer and Cause Host Anemia. Cell 2016, 164:246-257.

Bloodstream form trypanosomes release vesicles that can transfer cell contents to other parasites and cause phenotypic changes in vitro. The vesicles also fuse with host erythrocytes and alter their membrane properties.

9. DeJesus E, Kieft R, Albright B, Stephens NA, Hajduk SL: A single amino acid substitution in the group 1 Trypanosoma brucei gambiense haptoglobinhemoglobin receptor abolishes TLF-1 binding. PLoS Pathog 2013, 9:e1003317.

10. Matthews KR, McCulloch R, Morrison LJ: The within-host dynamics of African trypanosome infections. Philos Trans R Soc Lond B Biol Sci 2015, 370.

11. Vassella E, Reuner B, Yutzy B, Boshart M: Differentiation of African trypanosomes is controlled by a density sensing mechanism which signals cell cycle arrest via the cAMP pathway. J Cell Sci 1997, 110 ( Pt 21):26612671. 
12. Vassella $E$, Kramer $R$, Turner $C M$, Wankell $M$, Modes $C$, van den Bogaard $M$, Boshart M: Deletion of a novel protein kinase with PX and FYVE-related domains increases the rate of differentiation of Trypanosoma brucei. Mol Microbiol 2001, 41:33-46.

13. Domenicali Pfister D, Burkard G, Morand S, Kunz Renggli C, Roditi I, Vassella E: A Mitogen-activated protein kinase controls differentiation of bloodstream forms of Trypanosoma brucei. Eukaryot Cell 2006, 5:1126-1135.

14. Barquilla A, Saldivia M, Diaz R, Bart JM, Vidal I, Calvo E, Hall MN, Navarro M: Third target of rapamycin complex negatively regulates development of quiescence in Trypanosoma brucei. Proc Natl Acad Sci U S A 2012, 109:14399-14404.

15. ** Mony BM, MacGregor P, Ivens A, Rojas F, Cowton A, Young J, Horn D, Matthews K: Genome-wide dissection of the quorum sensing signalling pathway in Trypanosoma brucei. Nature 2014, 505:681-685.

A genome-wide screen for trypanosomes resistant to SIF mimetics identified many new components required for differentiation from slender to stumpy bloodstream forms.

16. Mony BM, Matthews KR: Assembling the components of the quorum sensing pathway in African trypanosomes. Mol Microbiol 2015, 96:220-232.

17. Brun $R$, Schönenberger M: Stimulating effect of citrate and cis-Aconitate on the transformation of Trypanosoma brucei bloodstream forms to procyclic forms in vitro. $Z$ Parasitenkd 1981, 66:17-24.

18. Dean S, Marchetti R, Kirk K, Matthews KR: A surface transporter family conveys the trypanosome differentiation signal. Nature 2009, 459:213-217.

19. ** Jones NG, Thomas EB, Brown E, Dickens NJ, Hammarton TC, Mottram JC: Regulators of Trypanosoma brucei cell cycle progression and differentiation identified using a kinome-wide RNAi screen. PLoS Pathog 2014, 10:e1003886.

A comprehensive RNAi screen in a variety of phenotypic assays.

20. Szöor B, Ruberto I, Burchmore R, Matthews KR: A novel phosphatase cascade regulates differentiation in Trypanosoma brucei via a glycosomal signaling pathway. Genes Dev 2010, 24:1306-1316.

21. Wenzler T, Schumann Burkard G, S. SR, Mäser P, Bergner A, Roditi I, Brun R: A new approach to chemotherapy: drug-induced differentiation kills African trypanosomes. Sci Rep 2016, 6:22451.

22. Muhich ML, Boothroyd JC: Polycistronic transcripts in trypanosomes and their accumulation during heat shock: evidence for a precursor role in mRNA synthesis. Mol Cell Biol 1988, 8:3837-3846.

23. Kelly S, Kramer S, Schwede A, Maini PK, Gull K, Carrington M: Genome organization is a major component of gene expression control in response to stress and during the cell division cycle in trypanosomes. Open Biol 2012, 2:120033. 
24. Güttinger A, Schwab C, Morand S, Roditi I, Vassella E: A mitogen-activated protein kinase of Trypanosoma brucei confers resistance to temperature stress. Mol Biochem Parasitol 2007, 153:203-206.

25. Droll D, Minia I, Fadda A, Singh A, Stewart M, Queiroz R, Clayton C: Posttranscriptional regulation of the trypanosome heat shock response by a zinc finger protein. PLoS Pathog 2013, 9:e1003286.

26.* Minia I, Clayton C: Regulating a Post-Transcriptional Regulator: Protein Phosphorylation, Degradation and Translational Blockage in Control of the Trypanosome Stress-Response RNA-Binding Protein ZC3H11. PLoS Pathog 2016, 12:e1005514.

The mechanism underlying the accumulation of $\mathrm{ZC} 3 \mathrm{H} 11$ in response to heat shock. This includes regulation of its own translation via an element in the $3^{\prime}$ UTR.

27.* Naguleswaran A, Gunasekera K, Schimanski B, Heller M, Hemphill A, Ochsenreiter T, Roditi I: Trypanosoma brucei RRM1 is a nuclear RNA-binding protein and modulator of chromatin structure. MBio 2015, 6:e00114.

TbRRM1 maintains permissive chromatin and acts as a nuclear effector of environmental temperature changes.

28. Vassella E, Van Den Abbeele J, Bütikofer P, Kunz Renggli C, Furger A, Brun R, Roditi I: A major surface glycoprotein of Trypanosoma brucei is expressed transiently during development and can be regulated post-transcriptionally by glycerol or hypoxia. Genes Dev 2000, 14:615-626.

29. Vassella E, Probst M, Schneider A, Studer E, Kunz Renggli C, Roditi I: Expression of a major surface protein of Trypanosoma brucei insect forms is controlled by the activity of mitochondrial enzymes. Mol Biol Cell 2004, 15:3986-3993.

30. Landeira D, Navarro M: Nuclear repositioning of the VSG promoter during developmental silencing in Trypanosoma brucei. J Cell Biol 2007, 176:133139.

31. Schumann Burkard G, Käser S, de Araujo PR, Schimanski B, Naguleswaran A, Knüsel S, Heller M, Roditi I: Nucleolar proteins regulate stage-specific gene expression and ribosomal RNA maturation in Trypanosoma brucei. Mol Microbiol 2013, 88:827-840.

32.** Fernandez-Moya SM, Carrington M, Estevez AM: A short RNA stem-loop is necessary and sufficient for repression of gene expression during early logarithmic phase in trypanosomes. Nucleic Acids Res 2014, 42:7201-7209.

The demonstration of a feedback loop between nucleoside availability and the stability of the mRNA encoding the transporter TbNT8. Once again, an element in 3' UTR functions as a sensor.

33. Oberholzer M, Lopez MA, McLelland BT, Hill KL: Social motility in African trypanosomes. PLoS Pathog 2010, 6:e1000739. 
34. * Imhof S, Knüsel S, Gunasekera K, Vu XL, Roditi I: Social motility of African trypanosomes is a property of a distinct life-cycle stage that occurs early in tsetse fly transmission. PLoS Pathog 2014, 10:e1004493.

Early and late procyclic forms are distinct entities, and have different biological properties. Only early procyclic forms have the capacity to perform SoMo.

35.** Oberholzer M, Saada EA, Hill KL: Cyclic AMP Regulates Social Behavior in African Trypanosomes. MBio 2015, 6:e01954-01914.

Chemical inhibition or depletion of a flagellar phosphodiesterase abolishes SoMo. The knockdown mutant can respond to exogenous migration factor, but is defective in its production.

36.* Lopez MA, Saada EA, Hill KL: Insect stage-specific adenylate cyclases regulate social motility in African trypanosomes. Eukaryot Cell 2015, 14:104-112.

Adenylate cyclases localized to the flagellar tip are negative regulators of SoMo. Trypanosomes encode more than 60 adenylate cyclases and this is a first indication that individual members are likely to have highly specialized functions.

37. Saada EA, Kabututu ZP, Lopez M, Shimogawa MM, Langousis G, Oberholzer $M$, Riestra A, Jonsson ZO, Wohlschlegel JA, Hill KL: Insect stage-specific receptor adenylate cyclases are localized to distinct subdomains of the Trypanosoma brucei flagellar membrane. Eukaryot Cell 2014, 13:1064-1076.

38. Imhof S, Vu XL, Butikofer P, Roditi I: A Glycosylation Mutant of Trypanosoma brucei Links Social Motility Defects In Vitro to Impaired Colonization of Tsetse Flies In Vivo. Eukaryot Cell 2015, 14:588-592.

39. Imhof S, Fragoso C, Hemphill A, von Schubert C, Li D, Legant W, Betzig E, Roditi I: Flagellar fusion and protein exchange in trypanosomes; a new form of cellcell communication. F1000Research 2016, in press.

40. Liniger M, Acosta-Serrano A, Van Den Abbeele J, Kunz Renggli C, Brun R, Englund PT, Roditi I: Cleavage of trypanosome surface glycoproteins by alkaline trypsin-like enzyme(s) in the midgut of Glossina morsitans. Int J Parasitol 2003, 33:1319-1328.

41. Kolev NG, Ramey-Butler K, Cross GA, Ullu E, Tschudi C: Developmental progression to infectivity in Trypanosoma brucei triggered by an RNAbinding protein. Science 2012, 338:1352-1353.

42. Burkard G, Fragoso CM, Roditi I: Highly efficient stable transformation of bloodstream forms of Trypanosoma brucei. Mol Biochem Parasitol 2007, 153:220-223. 
Legend to Figure 1

Overview of environmental factors sensed by different life-cycle stages of

Trypanosoma brucei. Some environmental cues modulate differentiation while others alter behavior. Parasite proteins that mediate responses are shown in boldface type. Slender bloodstream forms release stumpy induction factor (SIF) and exosomes (depicted as small circles) that are sensed by other parasites. To date, social motility and flagellar fusion have only been observed in vitro. 


\section{Mammalian host}

Serum components

Nutrients

Parasite components

\begin{tabular}{ll}
\multicolumn{2}{c}{ Insect host } \\
Temperature & Osmotic pressure? \\
Nutrients & Metabolites? \\
$\mathrm{pH}$ & lonic strength?
\end{tabular}

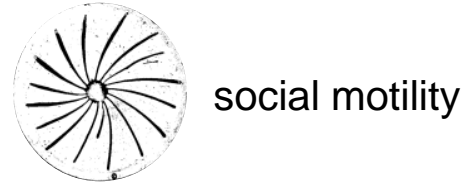

SIF

Kinases

RBP7

PPases
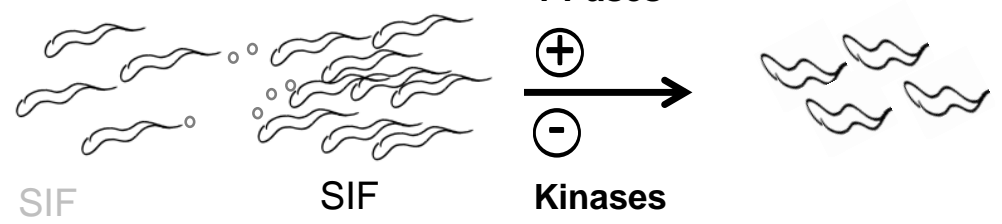

short stumpy

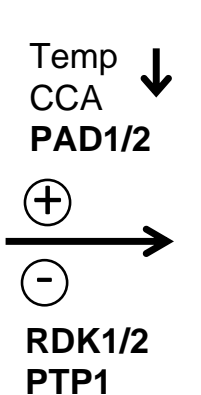

$\odot \oplus$

$\begin{array}{ll}\text { ACP1 } & \text { PDEB1 } \\ \text { ACP6 } & \text { RFT1 }\end{array}$

Glucose

Nucleolar flagellar fusion

$\widetilde{\widetilde{q}}$

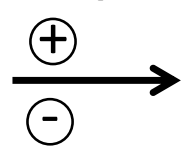

Glycerol long slender

early

late

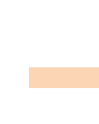

bloodstream forms

procyclic forms 